Document downloaded from:

http://hdl.handle.net/10251/146179

This paper must be cited as:

Del Castillo, RM.; Del Castillo, LF.; Calles, AG.; Compañ Moreno, V. (21-0). Experimental and computational study of conductivity of multilayer graphene in polypropylene nanocomposites. Journal of Materials Chemistry C. 6:7232-7241. https://doi.org/10.1039/c8tc01135d

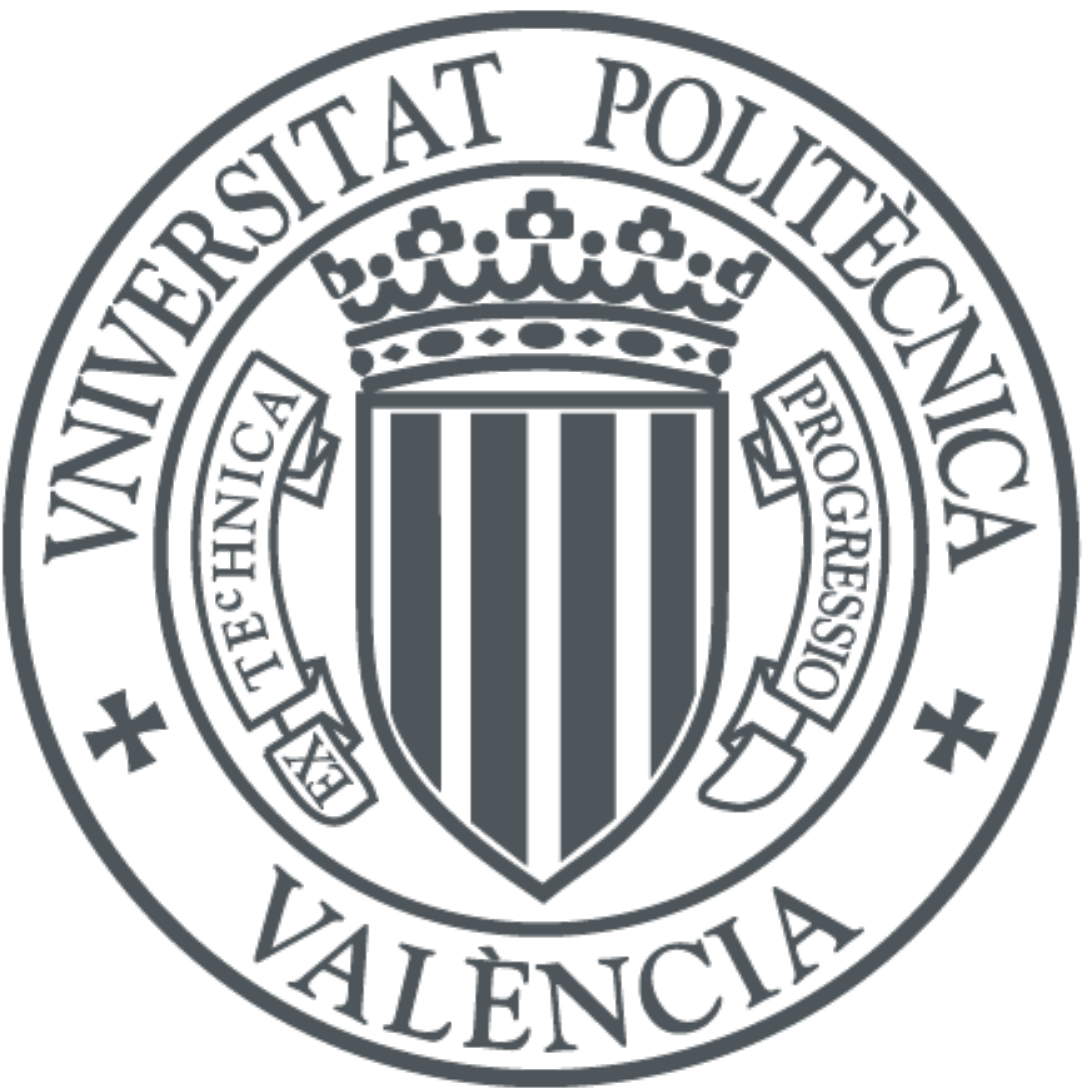

The final publication is available at

https://doi.org/10.1039/c8tc01135d

Copyright The Royal Society of Chemistry

Additional Information 


\section{Experimental and Computational Conductivity Study of Multilayer Graphene in}

\section{Polypropylene Nanocomposites.}

Roxana M. del Castillo ${ }^{1}$, Luis F. del Castillo², Alipio G. Calles ${ }^{1}$, Vicente Compañ ${ }^{3 *}$,

1. Departamento de Física, Facultad de Ciencias, Universidad Nacional Autónoma de México, Mexico City 04510, Mexico.

2. Departamento de Polímeros, Instituto de Investigaciones en Materiales, Universidad Nacional Autónoma de México, Apdo. Postal 70-360, Mexico City 04510, Mexico.

3. Departamento de Termodinámica Aplicada. Escuela Técnica Superior de Ingenieros Industriales (ETSII). Universidad Politécnica de Valencia, Campus de Vera s/n, 46020Valencia, Spain.

\section{Corresponding Author}

*E-mail address: vicommo@ter.upv.es (Vicente Compañ) 


\section{Abstract}

We study the electric conductivity of compounds formed by graphene multilayer in polypropylene. Our study makes a comparative analysis between experimental and computational results. To make an experimental measurement of the electronic properties, we deposited Multilayer Graphene (MLG) nanoparticles over a polypropylene matrix. The deposition was made in several stages, in which, we added to the polymer matrix different percentages of MLG nanoparticles using the melt compounding technique and we studied the conductivities of the nanocomposites by mean of electrochemical impedance spectroscopy (EIS). The second part consists of computational calculations, in which we studied the electronic properties of a graphene sheet under one polypropylene molecule with different degrees of polymerization. In both analysis, there is a strong percolation phenomenon with a percolation threshold around $18 \%$ of MLG nanoparticles. Before the percolation threshold, the charge carriers are constrained in the polypropylene molecule, making the system an insulating material and a p-type doping. After the percolation threshold, the charge carriers are constrained in the graphene, making the system a conductor material and n-type doping with conductivity values around $20 \mathrm{~S} / \mathrm{m}$. This phenomenon is a consequence of a change in the mechanism of charge transfer in the interface between polypropylene molecule and graphene sheet. In order to describe the charge transfer mechanism is necessary to consider the quantum effect. The incorporation of the quantum effects and the percolation phenomenon make possible for the theoretical conductivity to be close to the conductivity measured experimentally.

Keywords: Multilayer graphene; polypropylene; composite; conductivity; percolation. 


\section{Introduction}

Polypropylene is one of the most popular thermoplastic polymers, and it has many industrial applications. It is often used in the automobiles, textile, medical, and laboratory industry [1], due to its low density, rigidity, opacity and good stability at high temperatures. Propylene alone is an insulate material, but researchers have proven that polymers with a small number of nanoparticles as fillers enhance the physical properties of the material, like the thermal or electrical conductivity [2], the soundproofing ability and some mechanical properties [3]. Usually, the nanofillers chosen are multiwall carbon nanotubes [4], multilayer graphene, carbon nanofibers, and exfoliated graphite nanoplatelets [5]. The fact that the polymer changes its physical properties as the number of nanoparticles increases, obeys the percolation phenomenon, and there is a percolation threshold in the amount of nanoparticles in which the physical behavior changes drastically. It is well known that the geometric parameter called aspect ratio of the fillers is very important because the parameter determines the percolation threshold and the behavior of the interface between the polymer matrix and the fillers [6]. It has been observed that the percolation threshold is small in comparison with the number of nanofillers introduced in the system in polymers.

On the other hand, graphene has an extremely high conductivity, arriving to ballistic transition due to the high mobility of its charge carriers [7]. It has been seen that ballistic transport in graphene presents huge mean free paths for electrons, e.g., a graphene sheet grown by Chemical Vapor Deposition (CVD) technique presents a mean free path of $28 \mathrm{~mm}$ [8]. Evidently, the electrons in graphene behave as Dirac-fermions without mass, according to the previously reported [9], making graphene the most convenient filler to improve the 
electrical properties of composites. There are previous works with graphene as fillers in different types of polymers (epoxy, polystyrene, polyethylene, etc.) [10, 11]. These graphene fillers enhanced the physical properties at very low concentration of graphene nanoparticles in the polymer matrix.

According to previous studies, the percolation phenomenon has been seen from the experimental point of view and theoretically described with the Monte Carlo method. The classical point of view has predominated [12-14] in the description of the percolation, but we think that it is necessary to consider the quantum effects to represent correctly the interface. In the classical description of the percolation, it is usually assumed that the small percolation threshold of the graphene nanoparticles over a polymer matrix is a direct consequence of the hopping of the charge carriers [15], but there is much more to study about it.

Previous experiments with Multiwall carbon nanotubes (MWCNT) [4] and multilayer graphene (MLG) nanoparticles have been performed over a polypropylene matrix; microwave heating has been used to estimate the effects of the filler in the polymer matrix[16], taking advantage of the fact that the polymer matrix exhibits a low dielectric loss in the GHz region [17]. Carbon nanotubes are more susceptible to microwave radiation than graphene multilayers, proving that graphene multilayers could be a better filler than the carbon nanotubes [18]. In this work, we are studying the electrical conductivity from an experimental and a theoretical point of view. For the experimental point of view, a percentage of $0.5 \%, 1 \%, 3 \%, 5 \%, 10 \%, 15 \%, 20 \%, 25 \%$ and $30 \%$ of MGL nanoparticles were incorporated to the polypropylene matrix and then the conductivity was measured by electrochemical impedance spectroscopy (EIS). 
Regarding the theoretical descriptions in this work, we are using the previous description made by Weng et al.[15] but incorporating quantum effects into the aspect ratio. In particular, the quantum effects using electronic structure calculations to describe the ion transport phenomenon are being explored, and then these results are being used in a conductivity approach and compared with the experimental values. The electronic structure calculations were studied with the Density Functional Theory (DFT) and Quantum Espresso computational package.

\section{Methods}

\subsection{Sample preparation}

Multilayer graphene (MLG) was purchased from XGScience. Grade M with a diameter of 5 $\mu \mathrm{m}$ was selected for the trials, which is claimed to have high thermal and electrical properties. Homopolymer polypropylene (PP) was selected for the polymer matrix. Polypropylene was selected because it has a non-polar behavior, and thus; we can study the behavior of graphene nanoparticles in the polymer. The grade employed was PP DUCOR 1101S from DUCOR Petrochemicals. This material has an MFR $\left(230^{\circ} \mathrm{C} / 2.16 \mathrm{~kg}\right)$ of $25 \mathrm{~g} / 10 \mathrm{~min}$, a tensile modulus of $1500 \mathrm{MPa}$, and a melting point of $163^{\circ} \mathrm{C}$.

Nanocomposites with different percentages of MLG (PP+\%MLG) were obtained in a corotative twin screw extruder COPERION W\&P ZSK25. The extruder has a diameter of 25 $\mathrm{mm}$ and an L/D ratio of 40 . MLG nanocomposites were obtained with $0.5 \%, 1 \%, 3 \%, 5 \%$, $10 \%, 15 \%, 20 \%, 25 \%$ and $30 \%$ of filler loading. All the nanocomposites were produced with the same processing conditions. The fillers were incorporated via masterbatch produced in a previous process where MLG loading of $15 \%$ were produced under the following optimized conditions [16]: highly dispersive screw configuration, $600 \mathrm{rpm}$ and temperature profile 260 
${ }^{\circ} \mathrm{C} / 220{ }^{\circ} \mathrm{C} / 220{ }^{\circ} \mathrm{C} / 210{ }^{\circ} \mathrm{C} / 200{ }^{\circ} \mathrm{C} / 190{ }^{\circ} \mathrm{C}$. Masterbatch dilutions were processed with the same temperature profile with a screw speed of $800 \mathrm{rpm}$ and using a highly dispersive screw. Samples were obtained by compression molding in a hot press (COLLIN model $\mathrm{P} 200 \mathrm{E})$ at $200{ }^{\circ} \mathrm{C} / 15$ bars during $15 \mathrm{~min}$. Samples with dimensions of (10 x 1 x 0.4$) \mathrm{cm}$ were used to obtain the electrical conductivity using impedance spectroscopy electrochemical (ISE) studies [16].

\subsection{Dielectric properties}

The complex conductivity and permittivity of the compounds was measured by impedance spectroscopy at several temperatures within the $293 \mathrm{~K}\left(20^{\circ} \mathrm{C}\right)-333 \mathrm{~K}\left(60^{\circ} \mathrm{C}\right)$ range and frequency window $10^{-1}<f<10^{7} \mathrm{~Hz}$ using a Novocontrol Broadband Dielectric Spectrometer (Hundsangen, Germany) integrated with an SR 830 lock-in amplifier with an Alpha dielectric interface. The experiments were performed with $100 \mathrm{mV}$ amplitude. The samples PP+\%MLG were placed between two gold electrodes. During the conductivity measurements, temperature was kept isothermally or changed stepwise within the entire temperature range controlled by a nitrogen jet (QUATRO from Novocontrol) with a temperature error of $0.1 \mathrm{~K}$ during every single sweep in frequency.

\subsection{Computational Method}

To effectively describe the compounds proposed in this work, electronic structure calculations are being used. Graphene layer was designed as a 6x6 supercell, with a C-C bond length of $1.42 \AA$. The graphene layer is embedded in the $\mathrm{x}-\mathrm{y}$ plane, meanwhile the dimension of the supercell in the z-axis is large enough to neglect the effects between layers $(30 \AA)$. A degree of polymerization (PP) of the graphene sheet is made, i.e., over a graphene sheet, a polypropylene molecule with $N$ slabs $(N=1,5$, and 10) is added. The initial distance between 
the polypropylene molecule and the graphene sheet, at least between the hydrogen atoms, is $3 \AA$ A. Ground-state structure, adsorption energy and density of states (DOS) have been carried out with the Quantum-Espresso Computational Package [19], using a plane-wave set and pseudopotentials. Density Functional Theory (DFT) [20] was used with the generalized gradient approximation (GGA) and the Perdew-Burke-Ernzerhof parameterization (PBE) [21]. Kohn-Sham orbitals were expanded in a plane-wave basis-set up to a kinetic energy cutoff of 40 Ry. The convergence criterion for the self-consistent calculation was of $10^{-6} \mathrm{Ry}$, the Brillouin-zone integrations were carried out with the Methfessel-Paxton smearing technique [22], the smearing parameter was of $0.05 \mathrm{Ry}$. The Monksort-Pack approach [23] is being used to select the $k$-point mesh, with $\Gamma$-centered at $4 \times 4 \times 1$. The pseudopotentials for $\mathrm{C}, \mathrm{H}$, and $\mathrm{O}$ were chosen from the quantum espresso website [24]. All the pseudopotentials used the Vanderbilt approach [25]. The adsorption energy $\left(E_{a d s}\right)$ has been calculated according to the formula

$$
E_{\text {ads }}=E_{\text {graphene }+ \text { mol }}-\left(E_{\text {graphene }}+E_{\text {mol }}\right) .
$$

The $E_{a d s}$ is a measurement of the thermodynamic stability of the system. In equation 1 , $E_{\text {graphene+mol }}$ represents the total energy of the system formed by the molecule adsorbed into the graphene sheet, $E_{\text {graphene }}$ is the total energy of the graphene sheet, and $E_{m o l}$ is the energy of the polypropylene molecule in gas phase. If $E_{a d s}<0$, the adsorption process is exothermic, otherwise it is endothermic.

\section{Results}

Electrochemical impedance spectroscopy (EIS) measurements were carried out for all the samples of PP+\%MLG at different temperatures, $\left(20^{\circ} \mathrm{C}\right.$ to $\left.60^{\circ} \mathrm{C}\right)$, to obtain information on the samples conductivity. Data for the real part of the conductivity was analyzed in terms of 
the corresponding Bode diagrams, where variations of the conductivity with the frequency for all the composites of PP+\%MLG at different graphene concentrations $(\%=0.5,1,3,5$, 10,15,20, 25 and 30) are shown in figure 1. In this plot, we can see the double logarithmic plot of the conductivity in $\mathrm{S} / \mathrm{cm}$ versus frequency in $(\mathrm{Hz})$ at $50^{\circ} \mathrm{C}$ of temperature. Similar behavior was obtained for other temperatures, showing that composite samples are thermally inactive in all the range of temperatures studied, showing practically the same values of the conductivity for each one of temperatures. A close inspection of this figure shows that the real part of the conductivity for the samples in the range of 20 to $30 \%$ of MLG is constant for all range of frequencies. This behavior is the typical demeanor as a conductor material. However the conductivity is a function of the amount of fillers that we have incorporated in the nanocomposite. On the other hand, for PP+15\%MLG nanocomposite we observe that conductivity is practically constant in all the range of frequencies, only at frequencies higher than $10^{6} \mathrm{~Hz}$ the behavior of the sample shown a cut-off frequency where it starts increasing with the frequency. Similar observations we can see for the sample of PP+10\%MLG where the real part of the conductivity is also constant at the low frequencies region until a cut-off frequency where it starts increasing with the frequency, as if the sample were a capacitor. Finally, for the other samples with lower content of graphene oxide the plateau is not observed, presumably, because it is at very low frequencies, outside the range of measurement for our experiments, showing a dielectric behavior for the samples. The value of $\sigma$ constant means that the impedance has only a resistive contribution and its value represents the electrical conductivity of the sample. On the other hand, we have observe that the conductivity of the samples is practically independent of the temperature. The value of the conductivity of each one of nanocomposites can be obtained from the intercept in the 
OY-axis, (i.e. from the intersection of the extrapolated frequency-independent plateau line). Notice that in case of amount of fillers of $15 \%$ or minor, then in the high frequencies region the tendency is to change from the resistor to a capacitor behavior. The critical frequency is function of the amount of MLG fillers. For amounts of MLG below of $10 \%$ our results show straight lines with slope c.a. -1 , indicating that the nanocomposite at this concentrations are purely capacitors, where the values of the geometrical capacitance $\mathrm{C}$, for the samples of low content of MLG ( $\%=0.5,1,3,5)$, will be dependent of the amount of MLG incorporated into the matrix of polypropylene. Figure 1 shows that by increasing the amount of the MLG nanoparticles to the polypropylene film, the conductivity increases. The trend shows that by increasing the percentage of MLG nanoparticles over 15-20\%, the electrical conductivity will have the characteristic behavior of pure graphene [26]. 


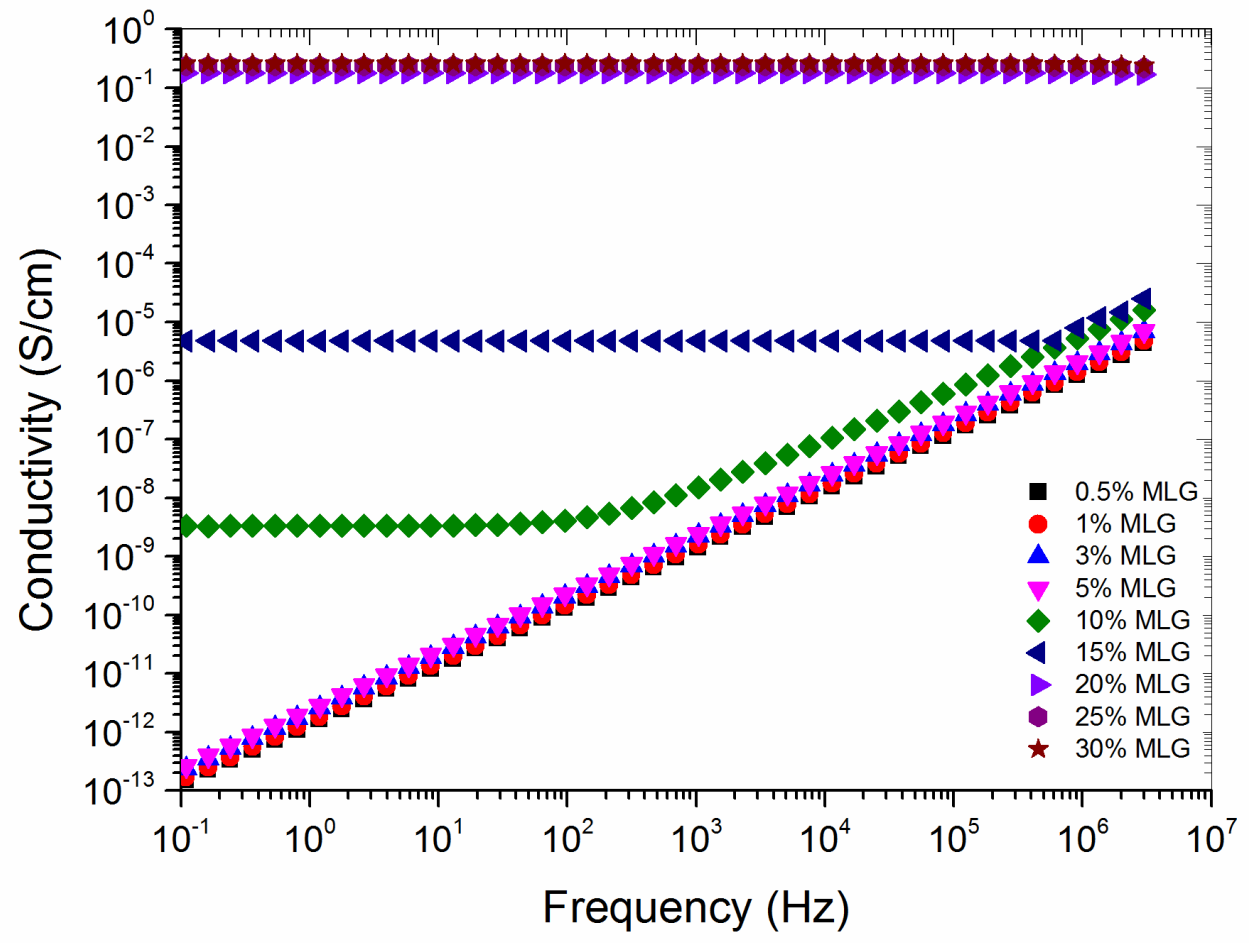

Figure 1. Double logarithmic plot of the real part of the conductivity as a function of the frequency for all the samples of PP+MLG at $50^{\circ} \mathrm{C}$. 


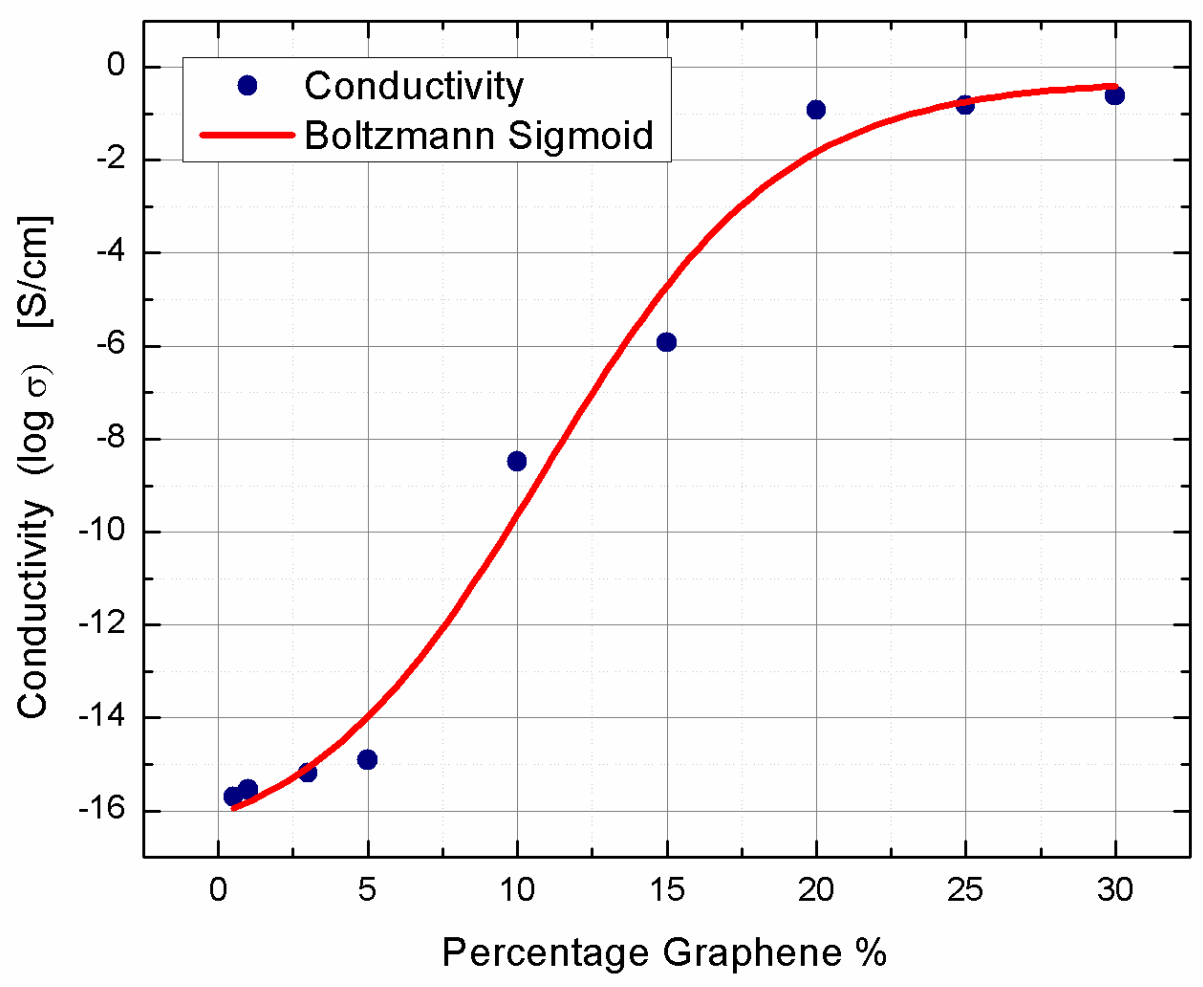

Figure 2. The experimental electrical conductivity versus the Percentage of MLG nanoparticles deposited in the polypropylene matrix.

Figure 2 shows the relationship between the conductivity and the percentage of MLG nanoparticles induced into the polypropylene matrix. It is possible to observe a percolation threshold around $18 \%$ of MLG nanoparticles fillers. Figure $\mathbf{2}$ was made using the experimental conductivity and then was fitted with the Boltzmann Sigmoid, according to the equation:

$$
y=\frac{-0.23521}{1+e^{(x-18.204 / 1.03592)}}-0.23521,
$$

With an error fit of $\mathrm{R}^{2}=0.905$. 
To accomplish a theoretical model of the conductivity of these nanocomposite materials we need to perform a complete study of the effects of interaction between the polypropylene matrix and the graphene [27]. Electronic structure calculations were carried out using a graphene supercell of 6x6 with adsorption of a polypropylene chain, which is formed with $N$ monomers as slabs $(N=1, N=5, N=10)$, the number of monomers included in the system indicates the degree of polymerization (PP). Each system was optimized according to the computational details to obtain the ground state structure or the optimized geometry of the system. Figure 3 shows the ground-state structures of the computational simulation for graphene-propylene $N=10$, graphene-propylene $N=5$, and graphene-propylene $N=1$.

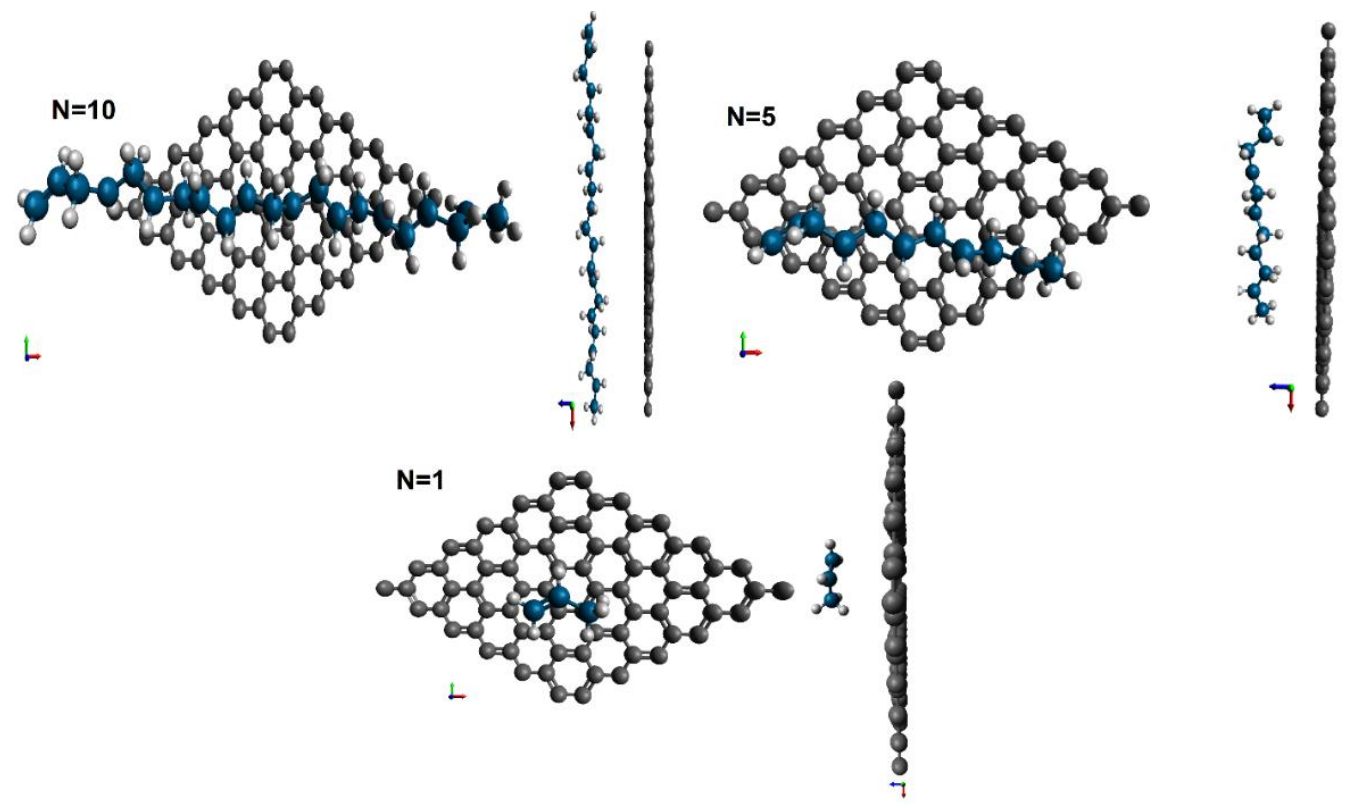

Figure 3. Ground-state structures of a polypropylene molecule $\left(C_{3} H_{6}\right)_{N}(N=10,5$, and 1), the value of $N$ indicates the degree of polymerization, adsorption on a graphene supercell (6x6), the carbons of the polypropylene molecule are shadowed in blue only for the purpose of distinguishing them from the carbons of the graphene sheet.

From Figure 3, it is possible to see the ground-state structure for the system graphenepolypropylene with three different degrees of polymerization. The polypropylene carbons are 
shadowed in blue only for the purpose of distinguishing them from the carbons of the graphene. The initial distance between the polypropylene axis and the graphene sheet (C-C) was $2.8 \AA$ A. However, after the geometry optimization, the polypropylene molecule moves away from the surface. Table 1 shows the bond length between the polypropylene and the graphene, also, it shows the adsorption energies of each system.

Table 1. Bond Length $(\AA)$ and adsorption energies (eV // kcal/mol) are presented for the graphene-polypropylene molecule with the degree of polymerization (PP).

\begin{tabular}{|c|c|c|c|}
\hline $\begin{array}{c}\text { PP of Graphene- } \\
\text { polypropylene } \\
(\mathbf{P P})\end{array}$ & Bond Length $(\mathbf{\AA})$ & $\begin{array}{c}\text { Adsorption energy }(\mathbf{e V} \\
\text { // kcal/mol) }\end{array}$ & \\
\hline $\mathrm{N}=1$ & & & 0.14 \\
\hline $\mathrm{N}=5$ & $4.29(2) / / 4.35$ & $-0.057 / /-1.32$ & 0.73 \\
\hline $\mathrm{N}=10$ & $4.28(6) / / 5.06(5)$ & $-1.81 / /-41.62$ & 1.55 \\
\hline
\end{tabular}

From the fact that the polypropylene molecule moves away from the graphene sheet and that the adsorption energies are very small, in the physisorption range, the Van der Waals forces are the only forces interacting in this adsorption process. Of the optimized geometries, it can be observed that the polypropylene molecule does not modify the graphene surface. Initially, the polypropylene film was chosen as the matrix for the deposition of MLG nanoparticles, because the polypropylene films present a non-polar behavior and this was thought to avoid the influence of the film on the MLG nanoparticle. From the simulations, it was observed that this assumption is appropriate, since the polypropylene moves away from the surface of graphene, minimizing the interactions between the polymer matrix and the MLG nanoparticles. By comparing the bond lengths of the separated compounds before and after the adsorption, we see that these bond lengths remain unchanged after the adsorption 
phenomena occurs. Because of the way in which the adsorption phenomenon happens (physisorption and bond lengths unchanged), we observe that there are two places where the charge carriers are confined; in the $p$-bonds of the polypropylene molecule; and the $\pi$-bonds of the graphene sheets. We concluded that before the percolation threshold, the charge carriers are trapped in the $p$-bonds of the polypropylene molecule, inhibiting the transport. Meanwhile, after the percolation threshold, the transport of the charge carriers is aquired through the $\pi$ bonds formed by the graphene sheet, improving transport.

We observed that introducing the MLG nanoparticles in the polypropylene matrix enhanced the conductivity of the polymer, transforming the polypropylene sheet from insulate to conductive material. It is possible to see that filling of the MLG nanoparticles in the polypropylene occurs as a non-linear function of the filler concentration. In fact, the filler concentration occurs following a percolation threshold which is influenced by several factors as aspect ratio of graphene sheets, inter-sheet junction, wrinkles and folds, etc [28].

For the sake of describing the interaction between the polypropylene matrix and the graphene nanoparticles, it is important to characterize the interface effects. The interface effect is a result of the interfacial charge carriers tunneling, enhancing the interfacial conductivity, which depends of the filler percentage $[6,15]$. When the filler percentage increases the average distance between fillers decreases, causing that the extra charge carriers move across the interface between the MLG nanoparticles and the polypropylene matrix. The electronic density of states (DOS) indicates how the charge carriers find the way to move. 

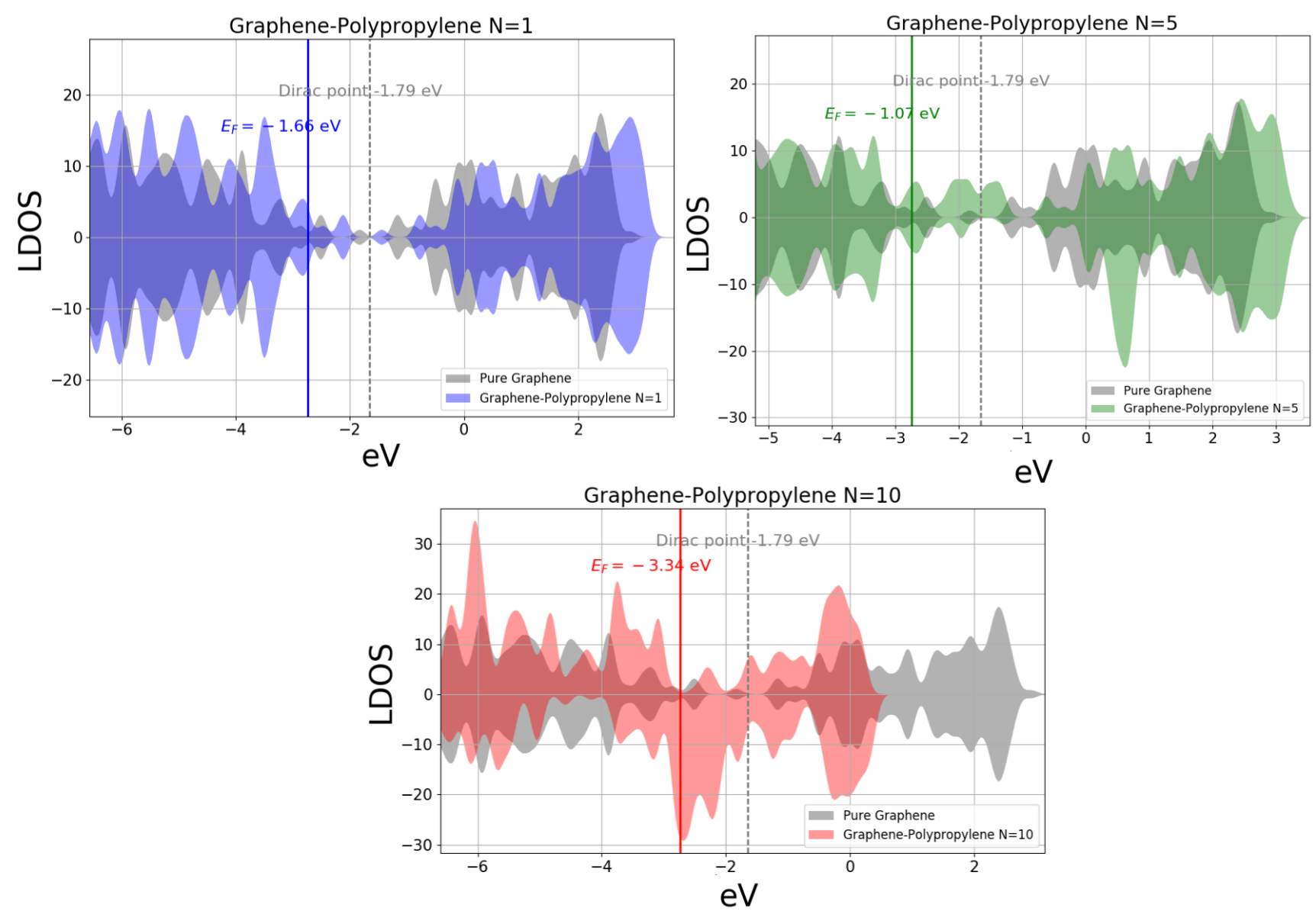

Figure 4. Electronic local density of states (LDOS) for pure graphene and graphenepolypropylene system.

The LDOS for pure graphene and graphene-polypropylene are shown in Figure 4. The grey line represents the LDOS of pure graphene and the blue/green/red line is the LDOS for graphene-polypropylene system with an $N$ of $\mathrm{PP}(N=1,5$, and 10$)$. It is well known that in weak disordered solids, the LDOS appears to be slightly different to the ideal case [29]. According to the adsorption energies, graphene-polypropylene systems are weak disordered crystals. In those systems in which the MLG nanoparticles have a predominant effect, after the percolation threshold, the Fermi energy $\left(E_{F}\right)$ of the graphene-polypropylene system ( $N$ $=1$, and $N=5$ ) is located at the right of the Dirac point, considering the Dirac point as the 
Fermi energy of pure graphene, remaining at the system as an $n$-type doped system. Following the description made by Leenaerts et al. [30], the charge transfer mechanism occurs from the polypropylene molecule to the graphene surface, because the localized states of the graphene-polypropylene system exceeds the Dirac point. Before the percolation threshold (graphene-polypropylene $N=10$ slabs), the polypropylene has the predominant effect, the LDOS tends to shift to the left of the LDOS of pure graphene. Regardless of the type of doping, increasing the amount of polymer in the system raises the energy gap, inhibiting the transport of the charge carriers. The $E_{F}$ is on the left side to the Dirac point, indicating that the system is $p$-type doped. The charge transfers from the graphene sheet to the polypropylene molecule. Therefore, we conclude that before the percolation threshold, when the material is insulated, there is very little transfer of charge and it goes from the graphene to the polypropylene matrix. After the percolation threshold is reached, the charge transfer mechanism changes, the charge goes from the polypropylene matrix to the graphene, which induces improved conductivity of the material.

Taking into account the classic descriptions made previously, conduction channels are induced when the nanoparticles are inserted randomly into the polymer matrix.We used one of these classic descriptions and incorporated quantum effects in the aspect ratio. In particular, we took the theoretical description made by Weng et al.[31, 32]. They modelled the percolation phenomenon of nanoparticles of graphene over a polymer matrix using the Eshelby's tensor [33, 34]. The Eshelby's tensor is a fourth order tensor $S_{i j k l}$, which describes the inclusion of a finite volume over a homogeneous matrix material. If the inclusion has an ellipsoidal shape, the Eshelby's tensor is reduced to a tensor of second order $S_{i j}$. In this work, the Eshelby's tensor is defined according to Landau and Lifshitz [35]: 


$$
S_{11}=S_{22}=\left\{\begin{array}{cc}
\frac{\alpha}{2\left(1-\alpha^{2}\right)^{\frac{3}{2}}}\left[\cos ^{-1} \alpha-\alpha\left(1-\alpha^{2}\right)^{\frac{1}{2}}\right], & \alpha<1 ; \\
\frac{\alpha}{2\left(\alpha^{2}-1\right)^{\frac{3}{2}}}\left[\alpha\left(\alpha^{2}-1\right)^{1 / 2}-\operatorname{arccosh} \quad \alpha\right], & \alpha>1 ; \\
S_{33}=1-2 S_{11} .
\end{array}\right.
$$

This definition of the Eshelby's tensor used a geometrical parameter defined as the aspect ratio $\alpha$, which is the thickness-diameter ratio of the fillers. With this definition of the Eshelby's tensor, it is possible to calculate $\left(c^{*}{ }_{1}\right)$ in the percolation threshold with the expression

$$
c_{1}=\frac{18 S_{11}^{3}-9 S_{11}}{18 S_{11}^{2}-3 S_{11}-4}
$$

Equation 3 is for low frequencies. To obtain the theoretical conductivity and the percolation threshold, determining how the graphene sheets are modified with the presence of the polypropylene molecules and the interface effects [7] is needed.

In previous works $[36,37]$, the formation of propagation channels from a statistical point of view, using the Cauchy statistical function to describe the tunneling is considered, and the most common computational treatment for this system is using the Monte Carlo simulation. In this work, the quantum effects are also considered. The quantum effects are taken into account in the electronic structure calculations and in the deformation measurement, as is shown in Figure 5. The interaction between the graphene sheet and the polypropylene molecule are described following the analysis discussed $\mathrm{in}^{38}$, the coordinates of the carbon atoms of the graphene sheet were plotted to obtain the deformation parameters, see Figure 5. The heights of the graphene sheet $(z)$, are taken as the fluctuation of the $z$-axis, considering 
that the graphene sheet, at the beginning, was placed in the plane $z=0$. The heights are shown in a blue-red scale. The deformation radius was taken as the distance between the maximum and minimum height near to the polypropylene molecule, see Figure 5. Another way to obtain the deformation radius is using the charge density surface (CDS). The aspect ratio was defined as the ratio $(\alpha)$ between the height $(z)$ and the deformation radius $(R), \alpha=\frac{z}{R}$.

Table 2. Deformation parameters from the graphene-polypropylene systems (height $z$ and radius $R$ ), the aspect ratio $\alpha$, the $S_{11}$ element of the Eshelby tensor, and $c^{*}{ }_{1}$ in the percolation threshold for each system.

\begin{tabular}{|c|c|c|c|c|c|c|}
\hline $\begin{array}{c}\text { PP of Graphene-polypropylene } \\
(N)\end{array}$ & $\begin{array}{c}z \\
(\AA)\end{array}$ & $\begin{array}{l}R \\
(\AA)\end{array}$ & $\alpha$ & $S_{11}$ & $c^{*}{ }_{1}$ & $\begin{array}{l}\text { Percolation } \\
\text { Threshold }\end{array}$ \\
\hline 1 & 0.26 & 4.01 & 0.064 & 0.472 & 0.171 & $17.1 \%$ \\
\hline 5 & 0.47 & 6.35 & 0.074 & 0.468 & 0.183 & $18.3 \%$ \\
\hline 10 & 0.349 & 4.62 & 0.054 & 0.476 & 0.185 & $18.5 \%$ \\
\hline
\end{tabular}

In the percolation threshold the conductivity behaves, predominantly, as graphene. The aspect ratio was defined as the ratio $(\alpha)$ between the height $(l)$ and the deformation radius $(R), \alpha=\frac{l}{R}=0.0468$, delivering a percolation threshold of $18.3 \%$ of MLG nanoparticle. The experimental measurement of $c^{*} 1$ in the percolation threshold is around $18 \%$ of MLG nanoparticles. 

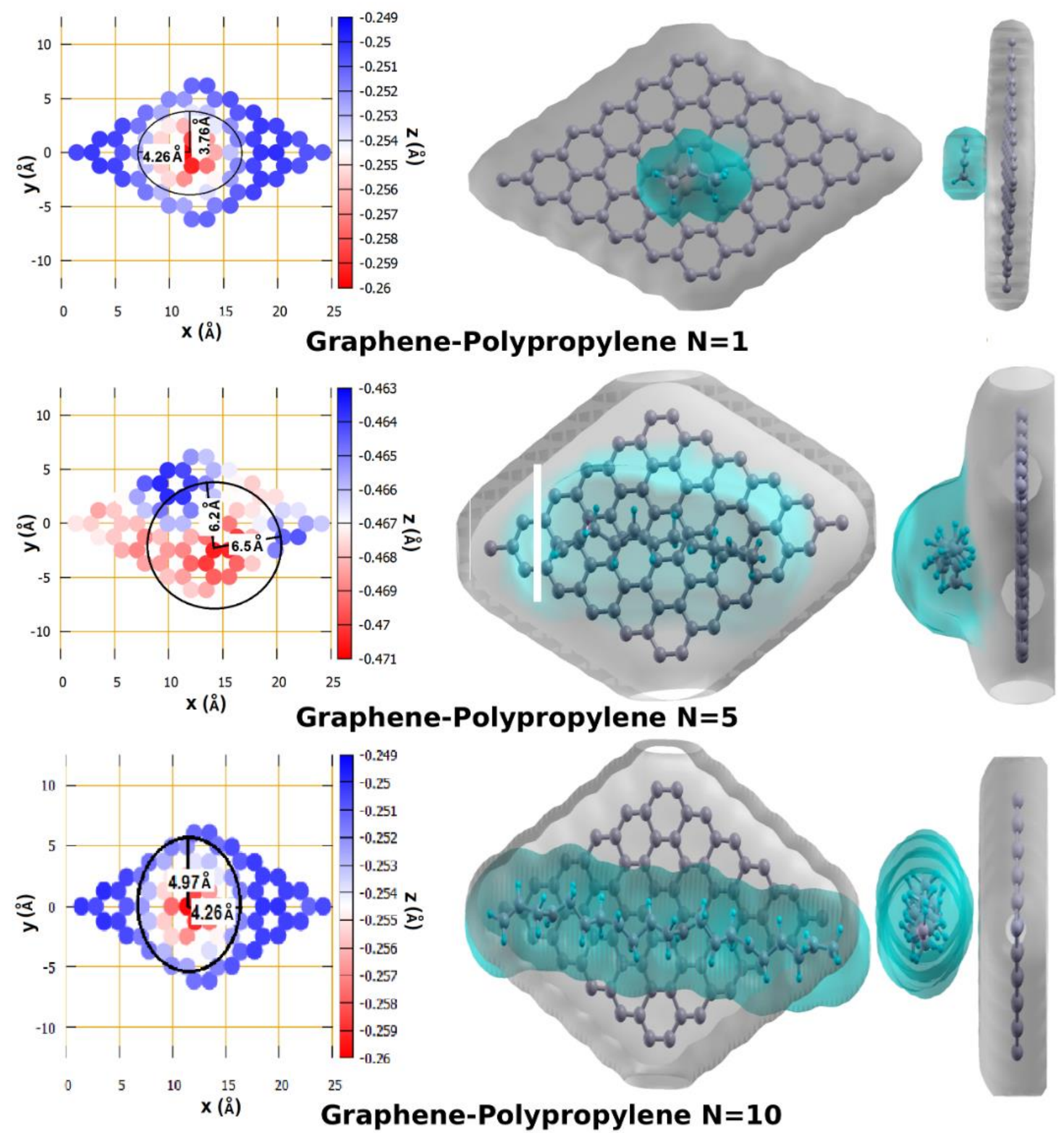

Graphene-Polypropylene $\mathbf{N}=10$

Figure 5. Optimized geometry of graphene-polypropylene molecule with $N$ of $\mathrm{PP}(N=1,5$, and 10). It shows the deformation of the graphene sheet produced by the polypropylene molecule with the coordinates and with the Charge Density Surface (CDS). 
Following previous studies about graphene conductivity [38], the theoretical conductivity of graphene can be estimated in terms of the elastic deformation of the graphene sheet. In our case, the conductivity measurement was made over the polypropylene matrix. After the percolation threshold the conductivity behaves as the conductivity of graphene doped with the polypropylene molecule. At this point, we are using the approach made by Wehling et al., which is:

$$
\sigma \approx\left(\frac{4 e^{2}}{\pi h}\right) \frac{n_{e}}{n_{i}} \ln ^{2}\left|\frac{E_{F}}{D}\right|
$$

In this approach, we are considering that the scattering mechanism is the midgap states [39]. In the Wehling's et al. paper, they deduced this conductivity formula in terms of the Green's function in two dimensions. $D$ is a parameter defined in terms of the nearest neighbor hopping $(t), D=\sqrt{\sqrt{3} \pi} t$, according to Wehling's work, it is possible to calculate $D$ in terms of the graphene deformation parameters $D=h v_{F} / R ; R$ is the deformation radius. From the optimization of the geometry ${ }^{40}$, it is viable to estimate $E_{F}$ of the graphene-polypropylene system, and $D$ as a function of $v_{F}=\sqrt{2 E_{F} / m_{e}} \quad\left(m_{e}=9.1 \times 10^{-31} \mathrm{~kg}\right.$ is the mass of the electron)[40]. $n_{i}$ is the number of impurities in $1 \mathrm{~cm}^{2}$; and $n_{e}$ is the number of charge carriers in $1 \mathrm{~cm}^{2}, n_{e}$ increases as the amount of MLG nanoparticles increases. The MLG nanoparticles are randomly deposited so at one minimum percentage of MLG (percolation threshold), charge carriers, and at least one conductive channel in which the charge carriers would flow through the polymer matrix will be found. The conductive and insulating regions will be combined, so that there will be thermodynamic fluctuation between each other, turning the insulation region into conductive areas. 
The terms of equation 4 are determined directly from the optimization of the geometry, except the fraction $n_{e} / n_{i}$. The fraction $n_{e} / n_{i}$ represents the fluctuation of charge carriers available in the system. When the system is insulated, there is no charge carriers available to be transported in the material, but, as soon the MLG nanoparticles are deposited in the system there is an exponential growth. The growth of charge carriers available to be transported is described by the following differential equation:

$$
\frac{d y}{d x}=\alpha y\left(1-\frac{y}{k}\right)
$$

we are using $y=n_{e} / n_{i}$, The solution to this differential equation is given by the logistic equation $\frac{n_{e}}{n_{i}}=\frac{k}{1+e^{d-\alpha x}}$. Again, following Weng et al. description ${ }^{31}$, the parameters of this logistic equation are given by the deformation of the system; $k$ is the asymptote value, given by the point of inflexion $k=24$; $d$ is the percolation threshold; $\alpha$ is the aspect ratio defined below as a geometrical parameter obtained directly from the simulation $(\alpha=0.049)$. 
Table 3. Estimation of the charge impurities ratio $n_{e} / n$, using the exponential growth model. Also, a direct comparison between the experimental conductivity and the theoretical conductivity is shown.

\begin{tabular}{|c|c|c|c|}
\hline$\% \mathrm{MLG}$ & $\boldsymbol{n}_{\boldsymbol{e}} / \boldsymbol{n}_{\boldsymbol{i}}$ & $\boldsymbol{\sigma}_{\text {teor }}(\mathbf{S} / \mathbf{c m})$ & $\boldsymbol{\sigma}_{\exp }(\mathbf{S} / \mathbf{c m})$ \\
\hline 0.5 & 0.00001 & $5.96 \mathrm{e}-12$ & $2.1 \mathrm{E}-16$ \\
\hline 1 & 0.00002 & $9.82 \mathrm{E}-12$ & $2.83 \mathrm{E}-16$ \\
\hline 3 & 0.00011 & $7.25 \mathrm{E}-11$ & $6.5 \mathrm{E}-16$ \\
\hline 5 & 0.00049 & $5.36 \mathrm{E}-10$ & $1.24 \mathrm{E}-15$ \\
\hline 10 & 0.02193 & $7.95 \mathrm{E}-8$ & $3.39 \mathrm{E}-9$ \\
\hline 15 & 0.94947 & 0.0112 & $1.23 \mathrm{E}-4$ \\
\hline 20 & 17.6014 & 0.209 & 0.18 \\
\hline 25 & 28.9656 & 0.237 & 0.225 \\
\hline 30 & 29.3901 & 0.237 & 0.24 \\
\hline
\end{tabular}

In Table 3, the $n_{e} / n_{i}$ parameters were calculated directly from the logistic equation. Then, these values were introduced into equation 4 and compared with the experimental conductivity. It looks like that the logistic equation is a good approach to describe the percolation problem with slight differences between the experimental and theoretical conductivity. The difference emerges before the percolation threshold, in which the composites behave as an insulator, because our theoretical approach is designed for composites with graphene. Figure 6 shows the direct comparison between the theoretical and the experimental sigmoid. The experimental sigmoid is being calculated in Figure 2, and obeys equation 2. The theoretical one is being fitted with the Boltzmann Sigmoid, according to the equation: 


$$
y=\frac{-0.23701}{1+e^{(x-17.997 / 0.99808)}}-0.23711
$$

With an error fit of $\mathrm{R}^{2}=0.96845$.

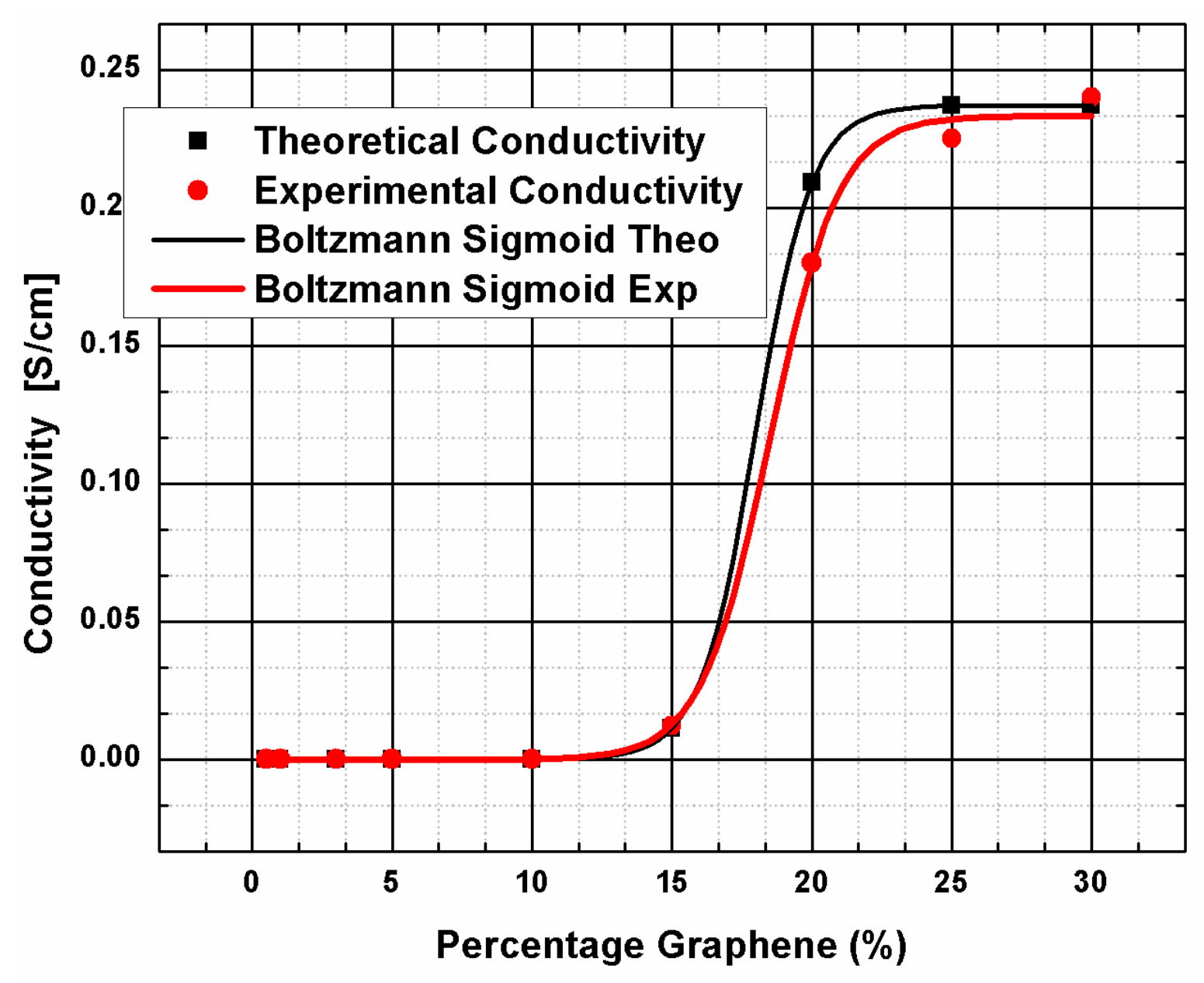

Figure 6.The theoretical conductivity versus the Percentage (\%) of MLG nanoparticles deposited in the polypropylene matrix (black color) is shown. On the other hand, in red color we show the experimental results for the same percentages of graphene. The lines show the sigmoid fits both experimental and theoretical results.

\section{Conclusions}

In this paper, we studied the compounds formed by graphene surfaces and polypropylene membranes. This study is divided in two parts; experimental and computational analysis. 
In both analysis, we observed that the conductivity curve can be fitted by a sigmoid curve, which is the main characteristic of the percolation phenomenon. The percolation threshold is around $18 \%$ of MLG nanoparticles. Likewise, the theoretical description of the percolation is based on the quantum framework, leading a more accurate description, very similar to the experimental results. In particular, the theoretical percolation threshold was calculated using the work of Weng et al.[34-36], using the fact that the percolation threshold depends only of a geometrical parameter (the aspect ratio $\alpha$ ). Now, in our present work, the aspect ratio parameter $\alpha$ was calculated after considering quantum effects in the geometry of the system, i.e., the optimization of the geometry was made by considering the quantum effects and this geometry was used to calculate the aspect ratio parameter $\alpha$, which gives us a percolation threshold very similar to the experimental threshold observed. With the percolation threshold, calculating the theoretical conductivity and comparing it with the experimental conductivity, is possible.

Secondly, from the adsorption energies we see, immediately, that the interactions between graphene and the polypropylene are Van der Waals type. This characteristic made possible to determine that, before the percolation threshold, the charge carriers are trapped in the $p$-bonds of the polypropylene molecule, restricting the transport. After the percolation threshold, the charge carriers are transported through the $\pi$-bonds of the graphene sheet. Furthermore, we observe that the geometry of the all molecules remained unchained, because the interaction between them is weak.

From the DOS, we see that increasing the number of slabs in the polypropylene molecule raise the energy gap making the system insulated. Also, we show that after the percolation threshold, the charge transfer mechanism is changed. Before the percolation threshold, the charge is transferred from the graphene sheet to the polypropylene molecule (this 
mechanism is not so efficient so the charge transport is very poor) $p$-type doping. After the percolation threshold, the charge is transferred from the polypropylene molecule to the graphene sheet $n$-type doping, improving the charge transport, turning the material into a conductive one.

The electrical conductivity was calculated using an approach made for pure graphene with resonant scattering [36]. This approach considered the quantum conductance owned by pure graphene and mid-gap states mechanism to describe the scattering of the charge carriers. The main parameters of this approach was calculated with DFT-theory in a geometry optimization. The number of charge carriers were determined based on the percolation phenomenon. When there is a high number of polypropylene molecules in the composite material, there are a low number of charge carriers. As soon as the MLG nanoparticles are introduced into the system, an exponential growth of the number of charge carriers in the composite material appears. This percolation phenomenon was introduced in the conductivity approach as the $n_{e} / n_{i}$ ratio (see equation 4 ). The theoretical conductivity estimate in this work is good enough compared to the experimental conductivity (see Table 2), taking into account the interface between the polypropylene molecule and the MLG nanoparticles.

\section{Acknowledgements}

This research has been supported by the ENE/2015-69203-R project, granted by the Ministerio de Economía y Competitividad (MINECO), Spain, Also Authors are grateful to UNAM-DGAPA-PAPIIT projects IG 100315, DGTIC-UNAM for access to the 
Miztli- UNAM supercomputer LANCAD-UNAM-DGTIC-055, and UNAM-DGAPA for the Postdoctoral grant of Roxana M. del Castillo.

\section{References}

[1]H.G. Karian. Handbook of polypropylene and polypropylene composites. RheTec, Inc. (2003) Second Edition. Whitmore Lake, Michigan. https://books.google.es/books?hl=es\&lr=\&id=C0nzeNPUpoIC\&oi=fnd\&pg=PP1\&dq=Handbook+o f+polypropylene+and+polypropylene+composites\&ots=LYqYBYg45n\&sig=3gtYXigr8_O8CUJeef BCtGI7QXA\#v=onepage $\& \mathrm{q}=$ Handbook $\% 20 \mathrm{of} \% 20$ polypropylene $\% 20 \mathrm{and} \% 20$ polypropylene $\% 20 \mathrm{c}$ omposites\&f=false

[2] T. Rath, Y., and Li. Nanocomposites Based on Polystyrene-B-Poly (Ethylene-R-Butylene)-BPolystyrene and Exfoliated Graphite Nanoplates: Effect of Nanoplatelet Loading on Morphology and Mechanical Properties. Composites Part A: Applied Science and Manufacturing, (2011), 42, 12, pp. 1995-2002.

http://www.sciencedirect.com/science/article/pii/S1359835X11002867

[3] M.S Kim, J. Yan, K.M. Kang, K.H Joo, Y.J. Kang, and S.H, Ahn. Soundproofing Ability and Mechanical Properties of Polypropylene/Exfoliated Graphite Nanoplatelet/Carbon Nanotube (PP/xGnP/CNT) Composite. International Journal of precision engineering and manufacturing (2013) 14, 6, pp. 1087-1092.

https://link.springer.com/article/10.1007/s12541-013-0146-3

[4] A. Rosehr, G.A. Luinstra. Polypropylene composites with finely dispersed multo-walled carbon nanotubes covered with an aluminium oxide shell.Polymer, (2017), pp. 120, 164-175.

http://www.sciencedirect.com/science/article/pii/S0032386117305220 
[5] D.D.L Chung. A review of exfoliated graphite. Journal of Material Science, (2016), 51, 1, 554568.

https://link.springer.com/article/10.1007/s10853-015-9284-6

[6] M. Terrones, O. Martín, M. González, J. Pozuelo, B. Serrano, J.C Cabanelas, S.M. Vega-Díaz, J. Baselga. Interphases in Graphene Polymer-based Nanocomposites: Achievements and Challenges.J., Adv. Mater, (2011), 23, 44, pp. 5302-5310.

http://onlinelibrary.wiley.com/doi/10.1002/adma.201102036/full

[7] K.I. Bolotin, K.J. Sikes, Z. Jiang, M. Klima, G. Fudenberg, J. Hone, P. Kim, H.L. Stormer. Ultrahigh electron mobility in suspended graphene. Solid State Commun, (2008), 146, 9-10. http://www.sciencedirect.com/science/article/pii/S0038109808001178

[8] L. Banszerus, M. Schmitz, S. Engels, M. Goldsche, K. Watanabe, T. Taniguchi, B. Beschoten, C. Stampfer. Ballistic Transport Exceeding $28 \mu \mathrm{m}$ in CVD Grown Graphene. Nano Lett., (2016), 16, 2, $1387-1391$.

http://pubs.acs.org/doi/abs/10.1021/acs.nanolett.5b04840

[9] B. Terrés, L.A. Chizhova, F. Libisch, J. Peiro, D. Jörger, S. Engels, A. Girschik, K. Watanabe, T. Taniguchi, S.V. Rotkin, J. Burgdörfer, C. Stampfer. Size quantization of Dirac fermions in graphene constrictions. Nature Communication,(2016), 7, 11528.

https://www.ncbi.nlm.nih.gov/pmc/articles/PMC4876454/

[10] S. Ansari, and E.P. Giannelis. Functionalized graphene sheet poly(vinylidene fluoride) conductive nanocomposites. J. Polym. Sci.Part B Polym. Phys, (2009), 47, 9, pp. 888-897.

http://onlinelibrary.wiley.com/doi/10.1002/polb.21695/full 
[11] H.B Zhang, W.B. Zheng, Q. Yan, Y. Yang, J.W. Wang, Z.H., Lu, G.Y. Ji, Z.Z Yu. Electrically conductive polyethylene terephthalate/graphene nanocomposites prepared by melt compounding. Polymer, (2010), 51, 5, pp. 1197-1196.

http://www.sciencedirect.com/science/article/pii/S003238611000056X

[12] K. Asadi, A.J. Kronemeijer, T. Cramer, L.J.A Koster, P.W.M Blom, and D.M. de Leeuw. Polaron hopping mediated by nuclear tunneling in semiconducting polymer at high carrier density. Nature Communications, (2013), 4; 1710.

https://search.proquest.com/docview/1349834005?pq-origsite=gscholar

[13] Z. Fan, F. Gong, S.T. Nguyen, and H.M. Duong, Advanced multifunctional graphene aerogelPoly(methyl methacrylate) composites: Experiments and modeling. Carbon, (2015), 81, pp. 396-404. http://www.sciencedirect.com/science/article/pii/S0008622314009245

[14] Z. Zabihi, and H. Araghi, Monte Carlo simulations of effective electrical conductivity of graphene/poly(methyl methacrylate) nanocomposite: Landauer-Buttiker approach. Synthetic Metals, (2016), 217, pp. 87-93.

http://www.sciencedirect.com/science/article/pii/S037967791630073X

[15] X. Xia, Z. Zhong, and G.J. Weng.Maxwell-Wagner-Sillars mechanism in the frequency dependece of electrical conductivity and dielectric permittivity of graphene-polymer nanocomposites. Mechanics of Materials, (2017), 109, pp. 42-50.

http://www.sciencedirect.com/science/article/pii/S0167663617300406

[16] T. Bayerl, A. Benedito, A. Gallegos, G.B Mitschang, and B. Galindo. Melting of PolymerPolymer Composites by Particulate Heating Promoters and Electromagnetic Radiation. Chap. 24 in 
Synthetic Polymer-Polymer Composites, by D Bhattacharyya, \& S. Fakirov, Carl HanserVerlag GmbH \& Co. KG, (2012), pp. 39-64.

http://www.hanser-elibrary.com/doi/abs/10.3139/9781569905258.002

[17] J. Harper, D. Price, and J. Zhang. Use of Fillers to Enable the Microwave Processing of Polyethylene. Journal of Microwave Power and Electromagnetic Energy, (2007), 219-227.

http://www.tandfonline.com/doi/abs/10.1080/08327823.2005.11688543

[18] B. Galindo, A. Benedito, E. Gimenez and V. Compañ. Comparative study between the microwave heating efficiency of carbon nanotubes versus multilayer graphene in polypropylene nanocomposites. Composites Part B, (2016), 98, pp. 330-338.

http://www.sciencedirect.com/science/article/pii/S1359836816305698

[19] P. Giannozzi, S. Baroni, N. Bonini, M. Calandra, R. Car, C. Cavazzoni, D. Ceresoli, G. Chiarotti, M. Cococcioni, et al. QUANTUM ESPRESSO: a modular and open-source software project for quantum simulations of materials. J. Phys.: Condens. Matter, (2009), 21, 9, 395502.

http://iopscience.iop.org/article/10.1088/0953-8984/21/39/395502/meta

[20] F.M. Bickelhaupt, and E.J. Baerends, Kohn-Sham Density Functional Theory: Predicting and Understanding Chemistry. In Reviews in Computational Chemistry, (2007), by Kenny B. Lipkowitz, \& Boyd Donald B., John Wiley \& Sons, Inc., 15, pp. 1-89.

http://onlinelibrary.wiley.com/doi/10.1002/9780470125922.ch1/summary

[21] J.P. Perdew, K. Burke, and M. Ernzerhof. Generalized Gradient Approximation Made Simple. Phys. Rev. Lett, (1996), 77, 3865.

https://journals.aps.org/prl/abstract/10.1103/PhysRevLett.77.3865 
[22] M. Methfessel, and A.T Paxton. High-precision sampling for Brillouin-zone integration in metals.Phys. Rev. B, (1989), 40, 3616.

https://journals.aps.org/prb/abstract/10.1103/PhysRevB.40.3616

[23] H.J. Monkhorst, J.D. Pack, Special points for Brillouin-zone integrations. Phys. Rev. B, (1976), 13.

https://journals.aps.org/prb/abstract/10.1103/PhysRevB.13.5188

[24]Files: C.pbe-van_ak.UPF, H.pbe-van_ak.UPF, N.pbe-van_ak.UPF, and O.pbe-van_ak.UPF.

http://www.quantum-espresso.org

[25] D. Vanderbilt. Soft self-consistent pseudopotentials in a generalized eigenvalue formalism. Phys. Rev. B, (1990), 41, 7892.

https://journals.aps.org/prb/abstract/10.1103/PhysRevB.41.7892

[26] K.S. Novoselov, A.K Geim, S.V. Morozov, D. Jiang, Y. Zhang, S.V. Dubonos, I.V. Grigorieva, A.A. Firsov. Electric Field Effect in Atomically Thin Carbon Films. Science, (2004), 306,5696, pp. 666-669.

http://science.sciencemag.org/content/306/5696/666

[27] R.M. Del Castillo, L.E. Sansores. Study of the electronic structure of Ag, Au, Pt and Pd clusters adsorption on graphene and their effect on conductivity. Eur. Phys. J. B, (2015), 88, 248, pp. 1-14.

https://link.springer.com/article/10.1140/epjb/e2015-60001-2

[28] D. Galpaya, M. Wang, M. Liu, M., N. Mottam, E. Waclawik, and C. Yan. Recent Advances in Fabrication and Characterization of Graphene-Polymer Nanocomposites. Graphene, (2012), 1,2, pp. $30-49$.

http://www.scirp.org/journal/PaperInformation.aspx?PaperID=23970 
[29] I. Zvyagin. Charge Transport via Delocalized States in Disordered Materials. In Charge Transport in Disordered Solids with Applications in Electronics, Ed. Sergei Baranovsky. John Wiley \& Sons, Inc, (2006), pp. 1-48, (2006).

http://onlinelibrary.wiley.com/doi/10.1002/0470095067.ch1/summary

[30] O. Leenaerts, B. Partoens, and F.M. Peeters. Adsorption of small molecules on graphene. Microelectronics Journal, (2009), 40, (4-5), pp. 860-862.

http://www.sciencedirect.com/science/article/pii/S0026269208005582

[31] R. Hashemi, and G.J. Weng, A theoretical treatment of graphene nanocomposites with percolation threshold, tunneling-assisted conductivity and microcapacitor effect in AC and DC electrical settings. Carbon, (2016), 96, pp. 474-490.

http://www.sciencedirect.com/science/article/pii/S0008622315303213

[32] X. Xia, Y. Wang, Z. Zhong, G.J. Weng, A frequency-dependent theory of electrical conductivity and dielectric permittivity for graphene-polymer nanocomposite. Carbon, (2017), 111, pp. 221-230.

http://www.sciencedirect.com/science/article/pii/S0008622316308387

[33] J.D. Eshelby. The determination of the elastic fiel of an ellipsoidal inclusion, and related problems. Proc. R. Soc. Lond. A, (1957), 241, pp. 376-396.

http://rspa.royalsocietypublishing.org/content/241/1226/376

[34] S. Trotta, F. Marmo, L. Rosati. Evaluation of the Eshelby tensor for polygonal inclusions. Composites Part B, (2017), 115, pp. 170-181.

http://www.sciencedirect.com/science/article/pii/S1359836816322132 
[35] L.D. Landau, E.M. Lifshitz, L.P. Pitaevskii, Electrodynamics of Continuous Media, Pergamon Press, New York, (1984), third ed.,

[36] Y. Wang, G.J. Weng, S.A. Meguid, A.M. Hamouda. A continuum model with a percolation threshold and tunneling-assisted interfacial conductivity for carbon nanotube-based nanocomposites. J. Appl. Phys. (2014), 115, 193706.

http://aip.scitation.org/doi/pdf/10.1063/1.4878195

[37] Y. Wang, J.W. Shan, G.J. Weng. Percolation threshold and electrical conductivity of graphenebased nanocomposites with filler agglomeration and interfacial tunneling. J. Appl. Phys. (2015), 118, 065101.

http://aip.scitation.org/doi/10.1063/1.4928293

[38] T.O. Wehling, S. Yuan, A.I. Lichtenstein, A.K. Geim, M.I. and Katsnelson. Resonant Scattering by Realistic Impurities in Graphene. Phys. Rev. Lett, (2010), 105, 056802.

https://journals.aps.org/prl/abstract/10.1103/PhysRevLett.105.056802

[39] T. Stauber, N.M.R. Peres, F. and Guinea. Electronic transport in graphene: A semiclassical. Physical Review B, (2007), 76, 205423.

https://journals.aps.org/prb/abstract/10.1103/PhysRevB.76.205423

[40] R.M. Del Castillo, and L.E. Sansores. Adsorption of Metal Clusters on Graphene and Their Effect on the Electrical Conductivity. In "Graphene Materials - Advanced Applications", (2017), by George Z Kyzas, \& Athanasios Ch. Mitropoulos. INTECH.

https://www.intechopen.com/books/graphene-materials-advanced-applications/adsorption-of-metalclusters-on-graphene-and-their-effect-on-the-electrical-conductivity 